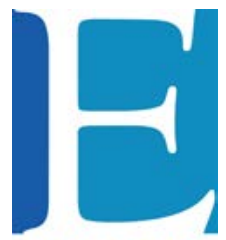

EVALUAR
2017, Vol. 17, No. 1

ISSN $1667-4545$

Recuperado de www.revistas.unc.edu.ar

Laboratorio de Evaluación Psicológica y Educativa Facultad de Psicología - Universidad Nacional de Córdoba

\title{
Propiedades psicométricas de una escala sobre el material artístico y su uso para resolver problemas en sesiones de terapia de arte
}

\author{
Psychometric properties of a scale about artistic material and its use to solve \\ problems in art therapy sessions
}

\author{
Minerva Vanegas-Farfano * 1 , Norma Cruz-Fierro ${ }^{2}$, Mónica González-Ramírez ${ }^{3}$ \\ 1. Universidad Autónoma de Nuevo León, UANL, Facultad de Psicología, México. \\ 2. Universidad Autónoma de Nuevo León, UANL, Facultad de Odontología, México. \\ 3. Universidad Autónoma de Nuevo León, UANL, Facultad de Psicología, México.
}

Introducción

Método

Resultados

Discusión

Referencias

Recibido: 27/01/2017 Revisado: 16/02/2017 Aceptado: 03/03/2017

\begin{abstract}
Resumen
El objetivo de este estudio fue generar un instrumento cuya validez y confiabilidad permitan evaluar el desarrollo de habilidades centradas en la solución de problemas mediante el uso de técnicas y material artístico dentro del espacio terapéutico. En una muestra de 216 participantes se validó una escala de 14 ítems con formato Likert. A partir del coeficiente alfa de Cronbach de .85 fue posible corroborar la consistencia interna de la escala, cuyos tres factores explican el $59.96 \%$ de la varianza total. No se encontraron diferencias significativas asociadas al género.
\end{abstract}

Palabras clave: arte y terapia, validación psicométrica, solución de problemas

\begin{abstract}
The objective of this study was to generate a reliable and valid scale that assessed the development of abilities focused on problem-solving through artistic techniques and materials on the therapeutic setting. On a 216 sample a 14 item Likert scale was evaluated. With a Cronbach's alpha's value of .85 , the resulting instrument reliability was corroborated; its three conforming factors explaining $59.96 \%$ of the total variance. No significant differences were found associated to gender.
\end{abstract}

Keywords: art and therapy, psychometric validation, problem-solving

\footnotetext{
*Correspondencia a: Minerva Vanegas-Farfano, minerva.vanegas@gmail.com, Universidad Autónoma de Nuevo León, UANL, Facultad de Psicología. Av. Universidad s/n, Ciudad Universitaria. San Nicolás de los Garza, Nuevo León, C.P. 66451. México.

Cómo citar este artículo: Vanegas-Farfano, M., Cruz-Fierro, N., \& González-Ramírez, M. (2017). Propiedades psicométricas de una escala sobre el material artístico y su uso para resolver problemas en sesiones de terapia de arte. Revista Evaluar, 17(1), 38-49. Recuperado de https://revistas.unc. 


\section{Introducción}

Actualmente la terapia psicológica se ha diversificado y entre sus opciones existen terapias que incluyen el uso de materiales artísticos como parte del tratamiento; algunas los utilizan como un apoyo, otras los consideran un aspecto central del modelo terapéutico. Si bien estos materiales median los resultados reportados en dichas intervenciones, existen pocas investigaciones enfocadas en su efecto específico (Kapitan, 2010; Malchiodi, 2005; Marxen, 2011; Spandler, Secker, Kent, Hacking, \& Shenton, 2007).

Conocer las razones por las que son útiles las herramientas que se utilizan es parte de la formación de todo profesionista, incluyendo el psicólogo clínico. Es por ello que la presente investigación se enfoca en el desarrollo de una evaluación para medir las propiedades formales y aquellas orientadas a los objetivos terapéuticos del uso de material artístico como apoyo en la terapia psicológica.

\section{El uso de materiales o desarrollo de objetos con objetivos terapéuticos}

El uso de materiales artísticos en espacios de rehabilitación tiene como objetivo valorar y recuperar habilidades físicas; sin embargo, aún sin tener una orientación a los aspectos psicológicos, de acuerdo a Alyami (2009) ayuda a afrontar problemas y disminuir síntomas depresivos, ansiosos o incluso el enojo derivado de percibirse vulnerable física o psicológicamente. A partir de hallazgos como el anterior es que se incrementa su empleo e investigación dentro del área de la salud psicológica.

Con independencia del enfoque teórico, el uso de técnicas y materiales se incluye en el desarrollo de diversas terapias (Marxen, 2011). De éstas, las denominadas terapias expresivas forman un grupo en donde tanto materiales como técnicas son entendidos como primordiales dentro de la clínica para gestar el cambio u objetivo deseado. Un ejemplo de ello es la terapia artística, la cual recurre a materiales gráficos para apoyar el bienestar físico, psicosocial o espiritual de la persona en momentos de crisis o tras un evento traumático (International Expressive Arts Therapy Association, s.f.; Johnson, 2009; Renton et al., 2012).

El uso de materiales artísticos, así como de las técnicas derivadas de la terapia artística, no sólo se encamina al apoyo de aquellos que cuentan con un diagnóstico clínico; estos elementos también se usan como parte de programas de prevención de la violencia (Tucker \& Treviño, 2011) o con personas que forman parte de programas comunitarios (Stacey \& Stickley, 2010). En otras palabras, se implementan con el objetivo de apoyar la salud, exista o no una situación clínica por resolver.

A pesar de la extensión en el uso de estos materiales dentro del campo de la salud, revisiones sistemáticas realizadas desde hace más de una década develan el desconocimiento en torno a por qué o cómo éstos ayudan a consolidar los objetivos terapéuticos (Reynolds, Nabors, \& Quinlan, 2000; Uttley et al., 2015). Contemplado desde distintos ángulos, dicho problema atrae la atención de asociaciones y profesionales por igual: no saber cuál es el aporte específico del material, cuestiona la necesidad de optar por éste ante la existencia de otras terapias donde no se requiere de una inversión extra (Stickley \& Hui, 2012). Por otro lado, existe desconocimiento acerca de si todos los pacientes deberían considerarse capacitados para utilizar materiales artísticos, o cómo habría de llevarse a cabo la evaluación de dichas aplicaciones para deslindarlas de, por ejemplo, la actividad propia del terapeuta (Feen-Calligan \& Nevedal, 2008). 


\section{La presente investigación}

Dentro del estudio y uso del arte con fines terapéuticos, los materiales artísticos son considerados capaces de dar forma a lo inasible; con el fin de dar o recobrar el sentido a las vivencias (Morales \& Jarpa, 2010). En la literatura sobre terapias expresivas, y especialmente sobre terapia artística, una parte de la investigación se concentra en dar respuesta a este interrogante, ya sea explorando la capacidad diagnóstica del dibujo (Betts, 2005; Gantt \& Anderson, 2009) o su posibilidad para dar cuenta del proceso o cambio terapéutico (Warson, 2003); y más recientemente para identificar qué aspectos evalúa el paciente al hablar de su uso. Dentro de este último grupo, destaca el proyecto elaborado por Snir y Regev (2013), quienes desarrollaron un instrumento de medición con la capacidad de valorar aspectos como los pensamientos y sentimientos que preceden a un proceso artístico, aquellos que ocurren durante éste, las actitudes hacia los objetos creados y el acercamiento hacia el material.

El estudio de Snir y Regev (2013) en el que se desarrolló el Art-based Intervention Questionnaire (ABI; Cuestionario sobre la Intervención Terapéutica Basada en Arte) fue realizado con 310 estudiantes universitarios dentro de un encuadre de taller creativo para evaluar el material. El cuestionario está conformado por 50 ítems divididos en cuatro dimensiones: sentimientos y pensamientos que preceden al proceso artístico (8 ítems), sentimientos y pensamientos que ocurren durante el proceso terapéutico (28 ítems), actitudes hacia la producción artística (6 ítems) y acercamiento a los materiales (8 ítems). Su validez convergente se evaluó implementando el Session Evaluation Questionnaire (SEQ; Stiles et al., 1994). Cabe mencionar que a la fecha no se han reportado investigaciones con resultados vinculados a su uso.

Contemplando que, más allá del cambio reportado en la sintomatología, es también ne- cesario descubrir de qué forma las actividades empleadas intervienen en estos cambios, este trabajo tuvo como objetivo construir y presentar evidencia sobre la validez y confiabilidad de una escala breve en idioma español diseñada a partir de los ítems del ABI.

\section{Método}

Participantes

Se formó una muestra de 216 adultos mexicanos (53 hombres y 162 mujeres), con una edad mínima de 18 y máxima de 22 años $(\mathrm{M}=19.88$; $\mathrm{DE}=4.17)$. Los participantes fueron convocados dentro de una facultad universitaria. Se realizó un muestreo aleatorio estratificado, contemplando tener la misma cantidad de alumnos de cada uno de los nueve semestres escolares con la finalidad de poder representar debidamente un grupo poblacional similar al examinado al momento de realizar el inventario original. La convocatoria se realizó dentro de la institución con apoyo del personal administrativo, quien proporcionó los datos de fechas y horarios en que podía acudirse a la institución para invitarles.

Se contempló como criterio de inclusión que el alumno se encontrase inscripto en al menos la mitad de las materias consideradas como de carga completa, que el posible participante se considerase capacitado para realizar actividades gráficas (dibujo) a nivel no profesional y que pudiese identificar una situación estresante que influyera en ese momento en su vida. Se consideró necesario excluir a alumnos que se encontrasen en período de duelo, tuvieran diagnóstico de ansiedad, depresión, estrés postraumático o problemas de abuso de sustancias. La participación fue voluntaria, anónima y confidencial y no se dieron créditos académicos ni ningún otro tipo de bonificación por colaborar en el estudio. 


\section{Instrumentos}

El instrumento evaluado, Actividad Gráfica como Herramienta (AGH), contempló para su construcción ítems del Art-based Intervention Questionnaire de Snir y Regev (ABI; 2013); autoinforme que, como ya se mencionó, se concentra en el proceso creativo experimentado en sesiones donde se utiliza material artístico. Las preguntas utilizadas del ABI fueron traducidas por el primer autor de este trabajo y analizadas previamente en un estudio piloto con 30 participantes, en el que la confiabilidad alcanzada fue de $\alpha=.76$.

Como ya ha sido mencionado, el ABI es un cuestionario cuya orientación para el entendimiento de las posibilidades terapéuticas se centra en los cambios ocurridos durante la actividad, mediante la inspección de la forma en que es experimentado el proceso creativo (Snir \& Regev, 2013). Dicha perspectiva, si bien permite conocer la reacción ante la experiencia creativa, no permite evaluar si ésta contribuye con el desarrollo de habilidades dentro del espacio terapéutico. Considerando que a la fecha la terapia artística no se enfoca en un tipo de dolencia particular, esta investigación incluyó el rediseño de preguntas orientadas al desarrollo de estrategias para solucionar problemas. Dicha elección obedece a la hipótesis de que una parte importante dentro de las terapias psicológicas es la búsqueda de que el paciente logre desarrollar habilidades que le permitan hacer frente a ciertos desafíos de manera adecuada (Chinaveh, 2012; Parto \& Besharat, 2011). Siguiendo este razonamiento, los ítems incluidos preguntan sobre estrategias cognitivas o comportamentales que pudiesen ayudar al individuo a cambiar la situación vivida o el significado de ésta. Son tres los ítems incluidos bajo esta perspectiva, y son el resultado de la modificación de tres preguntas pertenecientes al Inventario de Estrategias de Afrontamiento (CSI; Coping Strategies Inventory, por sus siglas en inglés), desde la versión validada para el idioma español por Cano-García, Rodríguez-Franco y García-Martínez (2007). Dicha escala fue realizada en su versión original por Tobin, Holroyd, Reynolds y Wigal (1989, en Cano-García et al., 2007) y mostró evidencias de validez convergente con el apoyo del Inventario de Personalidad NeoFFI. El CSI en su versión española incluye cuarenta ítems mediante los cuales se exploran los tipos de estrategias comunes ante estresores cotidianos (Cano-García et al., 2007). Dicha evaluación recoge datos tanto de forma cualitativa como cuantitativa mediante el empleo de una escala de tipo Likert. En este estudio, se utilizó exclusivamente la instrucción del componente cualitativo para dar pie a la intervención que habría de servir para evaluar el material artístico, como se señala en la sección de procedimiento.

En resumen, la escala cuyas propiedades psicométricas se evaluaron en esta investigación se conforma por 15 ítems con cinco opciones de respuesta tipo Likert, desde 0 (Nada) a 4 (Totalmente), orientados a medir el placer y valor terapéutico, la dificultad de uso del material y la capacidad con que cuenta éste para permitir la obtención de posibles soluciones. También se solicitó a los participantes que proporcionaran información concerniente a su edad, sexo y ocupación. En este último apartado se presentaron como opciones: "estudiante" y "estudiante y trabajador".

\section{Material}

Los materiales incluidos en este estudio para la evaluación de la AGH fueron: una hoja tamaño carta de papel Fabriano natural de bajo gramaje. Un paquete de crayones suaves tipo pastel de 12 piezas. Se eligieron por ser ampliamente utilizados en intervenciones de arte terapia, por su alta resistencia a la luz y por no requerir brochas o pinceles para su aplicación. También se entregaron un lápiz con borrador y elementos para limpiarse las manos. 


\section{Procedimiento}

Se acudió a las instalaciones de una institución educativa de nivel universitario donde fue solicitada la participación de los jóvenes. Los mismos fueron invitados a participar durante un día académico. Dada la cantidad de participantes $(\mathrm{N}=216)$, éstos fueron invitados en distintos horarios y días con la finalidad de no contaminar los resultados. Se señaló que la información sólo sería de conocimiento de los investigadores y se mantendría en anonimato el resultado de todos los documentos.

Cada sesión grupal incluyó un aproximado de treinta estudiantes. Al inicio de éstas se dio a conocer el propósito del estudio y aspectos relacionados con la participación: anonimato y voluntariedad, así como la necesidad de leer y completar la hoja de consentimiento. A todos los participantes se les entregó un sobre con los instrumentos de evaluación y material gráfico.

Dado que la finalidad de este estudio era valorar la capacidad del material artístico para el apoyo de los objetivos terapéuticos, fue necesario implementar una actividad que permitiese trabajar de una forma semejante a lo que sucede dentro del ámbito clínico. Fue así que, una vez llenado el consentimiento informado, se siguieron las instrucciones del componente cualitativo del CSI, las cuales solicitan al participante que piense en una situación estresante actual y la escriba. Dado que el objetivo del estudio es evaluar la capacidad del desarrollo de un dibujo bajo esas mismas condiciones, la instrucción se modificó para solicitar su representación mediante un dibujo. A fin de ampliar los conocimientos en torno a su uso como una forma pasiva de manejar el estrés (es decir, como un distractor) se solicitó a un subgrupo que representase un evento, objeto o persona que les ayudara a distraerse del problema o estresor. Posteriormente se pidió a todos los participantes completar los inventarios anteriormente descritos.
Análisis

Ya que el objetivo de este estudio era identificar el número y composición de los factores que componen al instrumento Actividad Gráfica como Herramienta $(\mathrm{AGH})$, cuya construcción contempló la inclusión de ítems de tres subescalas del ABI de Snir y Regev (2013), para llevarlo a cabo se tuvo como primer paso revisar la estructura subyacente de los ítems mediante un análisis factorial exploratorio (AFE; Lloret-Segura, Ferreres-Traver, Herández-Baeza, \& Tomás-Marco, 2014). Éste contempló como una primera opción utilizar el método de componentes principales con rotación varimax, tal y como fue realizado por los autores de la escala ABI (Snir \& Regev, 2013), de donde se retoman ítems pertenecientes a tres subescalas: placer y valor terapéutico, competencia, y dificultad para utilizar el material artístico. Buscando mantener la hipótesis de independencia entre variables señalada por dichos autores al construir esta escala, y la integración de ítems orientados al uso del material como estrategia para solucionar problemas (es decir, de un constructo a medir distinto a los anteriores), fue solicitada una solución de tres factores. Siguiendo las recomendaciones de Lloret-Segura et al. (2014) se contempló como criterio de retención de ítems que éstos presentaran pesos factoriales entre .40 y .70, condición moderada adecuada para escalas cuyos factores cuentan con 3 a 4 ítems revisados en muestras de 200 casos como mínimo.

Así mismo, considerando el sistema de medida ordinal empleado y la desestimación del análisis de componentes principales como análisis útil para la identificación del número y composición de factores, se realizó también un análisis bajo el método de mínimos cuadrados no ponderados. En este segundo análisis se solicitó tanto el gráfico de sedimentación como la extracción de los factores sugeridos por rotación oblicua. Cabe destacar que la pertinencia de efectuar el AFE se determinó tras obtener 
los índices de adecuación muestral, prueba de Kaiser-Meyer-Olkin y de esfericidad de Bartle$\mathrm{tt}$, considerando un valor superior a .80 como satisfactorio en la medida KMO (Lloret-Segura et al., 2014). La confiabilidad del instrumento, examinada en términos de consistencia interna de la escala general y subescalas, se evaluó mediante el estadístico alfa de Cronbach. En éste, se consideraron aceptables valores iguales o superiores a .70 (Spector, 1992). Se realizó también un análisis de correlaciones entre factores para, por un lado, asegurarse de la relación entre éstos y por otro descartar problemas de multicolinealidad. Para esto último se tuvo como criterio que el coeficiente de correlación r de Pearson no superase el valor de .90 (Field, 2016). Los anteriores análisis se llevaron a cabo mediante el paquete estadístico SPSS de IBM, versión 21 (2012).

Posteriormente se ejecutó un análisis factorial confirmatorio (AFC) que ayudó a verificar empíricamente los modelos sugeridos por el AFE. Sobre los modelos seleccionados se consideró pertinente que éstos: mantuviesen en cada factor un mínimo de 3 a 4 ítems, presentasen una saturación mínima de .40 y fuesen seleccionados tras contemplar los gráficos de sedimentación, siguiendo las recomendaciones de Lloret-Segura et al. (2014).

Considerando la configuración de ítems por factor, fueron comparados tres modelos: un primer modelo con sus factores relacionados, otro con un factor general de segundo orden y un tercero con tres factores de primer orden. En estos modelos se empleó el método de estimación de máxima verosimilitud. Para evaluar la bondad de ajuste se contempló: que tanto el índice de bondad de ajuste (GFI) como el índice comparativo de ajuste (CFI), el índice de bondad de ajuste regulado (AGFI) y el índice de Tucker-Lewis (TLI) alcanzaran valores iguales o mayores a .90. En el caso de la Chi cuadrada normada dividida entre los grados de libertad $\left(\chi^{2 / d f}\right)$, se verificó que su valor no excediese de 3; por último, en el caso de la raíz cuadrada media del error de aproximación (RMSEA) se verificó que su valor no superase a .80. Para establecer los anteriores valores se siguieron las recomendaciones de Byrne (2010). Para estos análisis se utilizó el software AMOS, versión 21 (2012), de IBM.

\section{Resultados}

A continuación, se presentan los datos correspondientes al AFE y AFC de la escala Actividad Gráfica como Herramienta (AGH).

\section{Construcción de la escala Actividad Gráfica como Herramienta (AGH)}

Se desarrolló un AFE utilizando el método de componentes principales con rotación varimax en SPSS v. 21. El resultado de la prueba de Bartlett fue $\chi^{2}=1343.80, p<.001$ y $\mathrm{KMO}=$ .88; dicho análisis indicó la existencia de una intercorrelación entre ítems adecuada para efectuar el análisis.

La prueba de sedimentación de Catell mostró la presencia de 3 factores tanto en el método de componentes principales como en mínimos cuadrados no ponderados. En el primero, la varianza total explicada fue del $59.96 \%$. Como se indicó en la sección de análisis, fueron considerados como pertenecientes a cada factor aquellos ítems que obtuviesen valores entre .40 y .70 en su carga. En la Tabla 1 se observan las cargas factoriales por ítem correspondientes a cada dimensión. Las cargas factoriales de las tres dimensiones se encuentran distribuidas en una sola columna y, como se puede observar, están dentro del rango propuesto o sobre éste, con excepción del ítem 14. En el caso del segundo ítem propuesto, éste fue borrado por mostrar un valor inadecuado, es decir, inferior a .30. En esta misma tabla, en las columnas tres a cinco, se expone la matriz de configuración co- 
rrespondiente al método de mínimos cuadrados no ponderados junto con la varianza explicada de cada factor, que en total explica el $50.79 \%$ de la varianza; $9.17 \%$ menos que lo encontrado por el método de componentes principales. Se muestran en itálicas los ítems cuyas cargas factoriales se observan en una dimensión distinta en el primer método, a saber, dos de los quince ítems (12 y 4).

Tabla 1

Pesos factoriales de la escala Actividad Gráfica como Herramienta (AGH).

\begin{tabular}{|c|c|c|c|c|}
\hline & $\begin{array}{c}\text { Peso } \\
\text { Factorial }^{\mathrm{a}}\end{array}$ & $\begin{array}{c}\text { Factor } \\
1^{\mathrm{b}} \\
34.64 \% \mathrm{c}\end{array}$ & $\begin{array}{c}\text { Factor } \\
2^{\mathrm{b}} \\
9.96 \% \mathrm{c}\end{array}$ & $\begin{array}{c}\text { Factor } \\
3^{\text {b }} \\
6.18 \% c\end{array}$ \\
\hline \multicolumn{5}{|l|}{ F1. Estrategia centrada en la solución de problemas } \\
\hline $\begin{array}{l}\text { 1. Te permitió distinguir estrategias para resolver el problema o situación } \\
\text { estresante. }\end{array}$ & .666 & .710 & & \\
\hline 3. Redobla tu esfuerzo para resolver la situación. & .686 & .701 & & \\
\hline 5. Te ayudó a hacerle frente al problema. & .744 & .775 & & \\
\hline 6. Te permite reconocer qué puedes hacer para que las cosas funcionen. & .745 & .784 & & \\
\hline 7. Apoya tu actual postura y el querer luchar por ella. & .712 & .784 & & \\
\hline 9. Tuviste la oportunidad de aprender algo sobre ti mismo(a). & .482 & .585 & & \\
\hline \multicolumn{5}{|l|}{ F2. Placer y valor terapéutico } \\
\hline 11. Sentiste agrado de hacer una actividad creativa. & .833 & & & .859 \\
\hline 15. Te permitió mejorar la confianza en ti. & .518 & & & .687 \\
\hline 10. Lograste concentrarte en tu paz interior. & .725 & & & .807 \\
\hline 12. Te dejaste llevar con el trabajo/actividad. & .770 & & .799 & \\
\hline \multicolumn{5}{|l|}{ F3. Competencia y uso del material } \\
\hline 4. Tuviste dificultades con el material para realizar lo que deseabas. & .786 & & & .773 \\
\hline 8. Presentaste dificultades para exponer (dibujar) tus ideas. & .598 & & .595 & \\
\hline 13. Te tomó tiempo entender cómo podías utilizar los materiales. & .579 & & .605 & \\
\hline 14. Pudiste permitirte cometer errores. & .367 & & .371 & \\
\hline
\end{tabular}

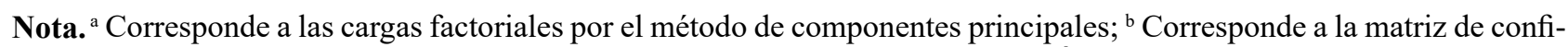
guración por método de mínimos cuadrados no ponderados. ${ }^{\mathrm{C}}$ Varianza explicada. Ítem eliminado: (2) Sabías exactamente cómo manejar el material.

La confiabilidad obtenida a nivel general y por subescalas se muestra en la Tabla 2. En la primera columna se observan los resultados por escala y subescala de acuerdo al método de componentes principales. En ésta la subescala Competencia y uso del material presenta un valor por debajo del $\alpha \geq .70$ recomendado. En la segunda columna se muestran los resultados contemplando el arreglo por mínimos cuadrados no ponderados; como se observa, existe una caída en la confiabilidad de las subescalas de Pla- cer y valor terapéutico y Competencia y uso del material.

Considerando que el grado de competencia depende del material utilizado y que únicamente fue valorada la técnica de pastel seco sobre papel, a diferencia del amplio grupo incluido por Snir y Regev (2013), se decidió retener los ítems de la subescala Competencia y uso del material pese a los resultados obtenidos tras utilizar el método de componentes principales. 
Tabla 2

Confiabilidad de la escala Actividad Gráfica como Herramienta (AGH).

\begin{tabular}{lcc}
\hline \multicolumn{1}{c}{ Subescala } & $\begin{array}{c}\boldsymbol{\alpha} \text { de Cronbach } \\
\mathbf{1 4} \text { ítems }^{\mathrm{a}}\end{array}$ & $\begin{array}{c}\boldsymbol{\alpha} \text { de Cronbach } \\
\mathbf{1 4} \text { ítems }\end{array}$ \\
\hline $\begin{array}{l}\text { Estrategia centrada en la } \\
\text { solución de problemas }\end{array}$ & .86 & .86 \\
$\begin{array}{l}\text { Placer y valor terapéutico } \\
\text { Competencia y uso del }\end{array}$ & .86 & .62 \\
$\begin{array}{l}\text { material } \\
\text { Escala General }\end{array}$ & .66 & .45 \\
\hline
\end{tabular}

Nota. $\left({ }^{a}\right)$ Factorización por el método de componentes principales. ( $)$ Factorización por el método de mínimos cuadrados no ponderados.

Por último, en la Tabla 3 se muestran las correlaciones interfactoriales correspondientes a la factorización de cada método: (a) para la factorización por el método de componentes principales y (b) para la factorización por el método de mínimos cuadrados no ponderados. Como se observa en el caso de la factorización por el método de componentes principales, existe una asociación fuerte entre la subescala de Estrategias centradas en la solución de problemas y el Placer y valor terapéutico, y ninguna relación entre las otras subescalas; mientras que en la factorización bajo el método de mínimos cuadrados no ponderados se encontraron asociaciones entre las tres subescalas, las cuales no superaron el valor de .90 recomendado por Field (2016).
La caída en la confiabilidad de las subescalas bajo el método de mínimos cuadrados no ponderados así como la disminución de la varianza total explicada por sus factores, en conjunto con la propuesta original del tipo de factorización a utilizar de acuerdo al cuestionario origen-modelo (Snir \& Regev, 2013) desestimó la ejecución de los siguientes análisis sobre el modelo de mínimos cuadrados no ponderados; por lo que el resto del escrito se concentra en el primer arreglo factorial.

Las medias y desviaciones estándar obtenidas por subescala y género se muestran en la Tabla 4. Estos valores se encontraron normalmente distribuidos en la subescala de Estrategias centradas en la solución de problemas $(\mathrm{Z}=$ $1.036, p=.234)$; Placer y valor terapéutico $(\mathrm{D}=$ $1.066, p=.205)$ y en la escala general $(\mathrm{Z}=.710$, $p=.695)$. Por otro lado, la escala de Competencia y uso del material presentó una distribución que no se ajustaba a la normalidad $(\mathrm{D}=2.402, p$ $<.001)$. La comparación por género de las puntuaciones por subescala y en el puntaje total de la escala no mostró diferencias significativas, dicho resultado se encuentra en la misma tabla.

Tabla 3

Correlaciones interfactoriales de la escala Actividad Gráfica como Herramienta (AGH).

\begin{tabular}{|c|c|c|c|c|c|c|}
\hline & $\mathbf{1}^{\mathrm{a}}$ & $\mathbf{1}^{\mathrm{b}}$ & $2^{\mathrm{a}}$ & $2^{\mathrm{b}}$ & $3^{\mathrm{a}}$ & $3^{\mathbf{b}}$ \\
\hline 1. Estrategia centrada en la solución de problemas & 1 & 1 & $.723 * *$ & $.607 * *$ & .094 & $.321 * *$ \\
\hline 2. Placer y valor terapéutico & & & 1 & 1 & .049 & $.606^{* *}$ \\
\hline 3. Competencia y uso del material & & & & & 1 & 1 \\
\hline
\end{tabular}

Nota. $\left({ }^{a}\right)$ Factorización por el método de componentes principales. $\left({ }^{b}\right)$ Factorización por el método de mínimos cuadrados no ponderados. $* * p<.001$ 
Tabla 4

Estadísticos descriptivos y comparación por sexo de las puntuaciones de la escala AGH.

\begin{tabular}{llccccccc}
\hline & $\begin{array}{c}\text { Estrategia centrada en la } \\
\text { solución de problemas }\end{array}$ & \multicolumn{2}{c}{$\begin{array}{c}\text { Placer y } \\
\text { valor terapéutico }\end{array}$} & \multicolumn{2}{c}{$\begin{array}{c}\text { Competencia y uso del } \\
\text { material }\end{array}$} & \multicolumn{2}{c}{$\begin{array}{c}\text { Actividad Gráfica como } \\
\text { Herramienta (AGH) }\end{array}$} \\
\hline & $\mathrm{M}$ & $\mathrm{DE}$ & $\mathrm{M}$ & $\mathrm{DE}$ & $\mathrm{M}$ & $\mathrm{DE}$ & $\mathrm{M}$ & $\mathrm{DE}$ \\
Hombres & 12.43 & 5.76 & 8.47 & 4.69 & 4.83 & 3.45 & 25.73 & 10.91 \\
Mujeres & 11.97 & 5.75 & 9.22 & 4.24 & 5.03 & 3.24 & 26.22 & 9.98 \\
$\mathrm{~N}=216$ & 12.08 & 5.74 & 9.03 & 4.36 & 4.98 & 3.29 & 26.10 & 10.19 \\
Prueba $t$ & $t=.504, p=.615$ & $t=1.08, p=.279$ & $t=.372, p=.711$ & $t=.304, p=.761$ \\
\hline
\end{tabular}

Nota. $\mathrm{M}=$ media; $\mathrm{DE}=$ desviación estándar.

\section{Análisis factorial confirmatorio}

El AFC se realizó con las respuestas de la misma muestra. Los valores a analizar corresponden al modelo de tres factores y 14 ítems. Se seleccionaron seis estadísticos para evaluar la bondad de ajuste de dicho modelo hipotético: Chi cuadrada normada dividida entre los grados de libertad $\left(\chi^{2} / d f\right)$; la raíz cuadrada media del error de aproximación (RMSEA); el índice de ajuste comparativo de Bentler (CFI); el índice de bondad de ajuste (GFI); el índice de bondad de ajuste regulado (AGFI) y el índice de Tucker-Lewis (TLI). Fueron tomados como criterios de ajuste adecuado que la razón entre Chi cuadrada y los grados de libertad no exceda de 3, que la RMSEA no supere un valor de $.08 \mathrm{y}$ que los índices CFI, GFI, AGFI y TLI presen- ten valores cercanos a .90 (Byrne, 2010). Los resultados obtenidos se muestran en la Tabla 5. En el primer modelo, con tres factores de primer orden, únicamente el AGFI presentó resultados por debajo del valor considerado pertinente, sin embargo, se acercaba al límite recomendado, por lo que se consideró que presentaba un buen ajuste. Los valores del segundo modelo revisado, con un factor general de segundo orden, se muestran en la misma tabla, en este modelo ninguno de los valores se encontró dentro de los considerados aceptables, por lo que fue descartado. Por último, en el caso del modelo de tres factores de primer orden, éste presentó valores semejantes a los del primer modelo, con un ligero incremento en el GFI (.912), ubicándose como el mejor de los tres modelos. La Figura 1 muestra la escala resultante.

Tabla 5

Estadísticos de bondad de ajuste.

\begin{tabular}{|c|c|c|c|c|c|c|}
\hline Modelo & $\chi^{2 / d f}$ & RMSEA & CFI & GFI & AGFI & TLI \\
\hline 1. Tres factores relacionados & 1.987 & .068 & .940 & .911 & .875 & .927 \\
\hline 2. Factor general de segundo orden & 5.152 & .139 & .741 & .750 & .659 & .694 \\
\hline 3. Tres factores de primer orden & 1.996 & .068 & .940 & .912 & .875 & .927 \\
\hline
\end{tabular}




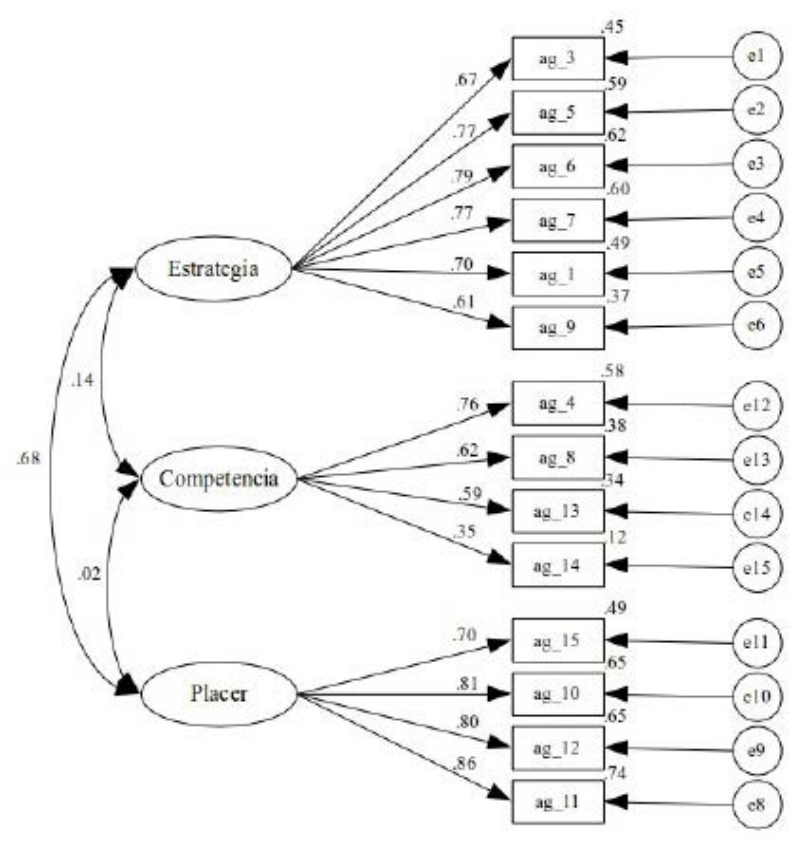

Figura1

Estructura factorial de la AGH.

Nota. Se presentan los valores estandarizados.

\section{Discusión}

Este estudio tuvo como objetivo el diseño y análisis dimensional de una escala enfocada en la evaluación del uso de material artístico con fines terapéuticos. Su construcción incluyó tanto la revisión de trabajos similares en otro idioma, como la indagación de los aspectos que pudiesen implementarse para evaluar las propiedades singulares que le distinguen, más allá de la intervención terapéutica. La escala resultante (Actividad Gráfica como Herramienta, AGH) cuenta con tres dimensiones, las cuales permiten evaluar la posibilidad del material artístico para fungir como medio para generar alternativas, redoblar esfuerzos o aprender de sí mismo (Estrategia centrada en la solución de problemas), reflexionar sobre la actividad y proceso creativo, lograr una mayor confianza y paz (Placer y valor terapéutico) y por último, conocer la propia capacidad para manejar el material (Competencia y uso del material). Su confia- bilidad general es buena $(\alpha=.85)$; con niveles semejantes en dos de sus tres componentes $(\alpha=$ .86 , Estrategia centrada en la solución de problemas; $\alpha=.86$, Placer y valor terapéutico); y un valor cercano al .70 aceptable en la escala de Competencia y uso del material $(\alpha=.66)$.

Para la construcción de la AGH se consideró fundamental el revisar escalas con objetivos semejantes. Al sólo ser posible encontrar este tipo de material en inglés (ABI; Snir \& Regev, 2013), esta investigación tuvo que incluir tanto el desarrollo de un AFE, para identificar la existencia de un modelo cuya configuración se asemejase al modelo de habla inglesa; como su posterior comprobación mediante un AFC. En este último análisis, tres modelos trifactoriales fueron analizados intentando con ello encontrar el mejor de éstos. Tras realizar este análisis se obtuvo un modelo consistente con los fundamentos teóricos, de construcción e hipotéticos derivados del cuestionario realizado por Snir y Regev (2013); pero ampliado en relación a las estrategias para la solución de problemas.

Esta escala se suma a los pocos instrumentos diseñados para evaluar las propiedades del material y la actividad artística que pueden relacionarse con el trabajo y la mejora en la salud psicológica. Según nuestro conocimiento, es la primera desarrollada en idioma español. Es también el primer estudio que evalúa, aunque parcialmente, las propiedades psicométricas del ABI (Snir \& Regev, 2013). Es además una escala que, dado el procedimiento utilizado para desarrollarla -sin la intervención directa de un terapeuta-, permite distinguir sus propiedades más allá del objetivo y el rol del terapeuta, algo señalado como necesario en el desarrollo del campo de la arte terapia y terapias expresivas (Reynolds et al., 2000; Muri, 2007).

Como se señaló, dentro de la arte terapia y terapias expresivas, con independencia del enfoque teórico, muchas terapias incluyen en su ejecución el empleo de materiales como parte de la intervención. Siguiendo esta forma 
de trabajo la AGH incorpora la evaluación del material artístico en función de sus propiedades terapéuticas haciendo únicamente un énfasis en la posibilidad de introspección y generación de alternativas.

\section{Limitaciones y estudios a futuro}

Dentro de las limitantes de este estudio es necesario mencionar que en la construcción de la AGH únicamente se evaluó el empleo de un tipo de material, esto se vio reflejado en la baja confiabilidad de una de las dimensiones, por lo que se recomienda ampliar las investigaciones con este instrumento pero utilizando otros materiales, al igual que su empleo con otras poblaciones.

Asimismo se recomienda su evaluación siguiendo distintos formatos de intervención, es decir, contemplando no sólo su empleo para el trabajo con situaciones estresantes cotidianas sino también con otros temas como el estrés postraumático o el manejo del duelo, por mencionar algunos. Se recomienda también su revisión en estudios que incluyan más de una sesión.

\section{Referencias}

Alyami, A. (2009). The integration of art therapy into physical rehabilitation in a Saudi hospital. The Arts in Psychotherapy, 36(5), 282-288. doi: 10.1016/j. aip.2009.07.001

Betts, D. (2005). A systematic analysis of art therapy assessment and rating instrument literature. (Tesis doctoral). Recuperado de http://diginole.lib.fsu. edu/islandora/object/fsu:175923

Byrne, B. (2010). Structural equation modeling with AMOS. Basic concepts, applications, and programming. NY: Routldege.

Cano-García, F. J., Rodríguez-Franco, L., \& García-Martínez, J. (2007). Adaptación española del Inventario de Estrategias de Afrontamiento. Acta
Española de Psiquiatría, 35(1), 29-39. Recuperado de http://personal.us.es/fjcano/drupal/files/ AEDP $\% 2007 \% 20$ (esp).pdf

Chinaveh, M. (2012). The effectiveness of problem-solving on coping skills and psychological adjustment. Procedia - Social and Behavioral Sciences, 84, 4-9. doi: 10.1016/j.sbspro.2013.06.499

Feen-Calligan, H., \& Nevedal, D. (2008). Evaluation of an art therapy program: Client perceptions and future directions. Art Therapy, 25(4), 177-182. doi: 10.1080/07421656.2008.10129544

Field, A. (2016). An Adventure in Statistics: The Reality Enigma. Thousand Oaks, CA: Sage.

Gantt, L. M., \& Anderson, F. (2009). The Formal Elements Art Therapy Scale: A measurement system for global variables in art. Art Therapy, 26(3), 124129. doi: 10.1080/07421656.2009.10129372

IBM (2012). SPSS Statistics 21.0.0. [software de cómputo]. Recuperado de https://www-01.ibm.com/ software/mx/analytics/spss/products/statistics/

IBM (2012). SPSS AMOS 21.0.0 [software de cómputo]. Recuperado de http://www.spss.com.hk/amos/index.htm?tab=1

International Expressive Arts Therapy Association. (s.f.). What we do? Recuperado el 17 de Noviembre de 2016 de http://www.ieata.org/what-we-do.html

Johnson, D. R. (2009). Commentary: Examining underlying paradigms in the creative arts therapies of trauma. The Arts in Psychotherapy, 36(2), 114120. doi: 10.1016/j.aip.2009.01.011

Kapitan, L. (2010). Introduction to Art Therapy Research. NY: Routledge.

Lloret-Segura, S., Ferreres-Traver, A., Hernández-Baeza, A., \& Tomás-Marco, I. (2014). El análisis factorial exploratorio de los ítems: Una guía práctica, revisada y actualizada. Anales de Psicología, 30(3), 1151-1169. doi: 10.6018/analesps.30.3.199361

Malchiodi, C. A. (Ed.). (2005). Expressive Therapies. Nueva York, NY: Guilford Publication.

Marxen, E. (2011). Diálogos entre arte y terapia. Del "arte psicótico" al desarrollo de la arteterapia y sus aplicaciones. Barcelona, España: Gedisa.

Morales, P., \& Jarpa, J. M. (2010). Elementos de Arteterapia como medio de comunicación y elaboración 
en psicoterapia con adolescentes. Arteterapia: $\mathrm{Pa}$ peles de arteterapia y educación artística para la inclusión social, 5, 137-152. Recuperado de http:// revistas.ucm.es/index.php/ARTE

Muri, S. A. (2007). Beyond the face: Art therapy and self-portraiture. The Arts in Psychotherapy, 34(4), 331-339. doi: 10.1016/j.aip.2007.05.002

Parto, M., \& Besharat, M. A. (2011). The direct and indirect effects of self-efficacy and problem-solving on mental health in adolescents: Assessing the role of coping strategies as mediating mechanism. Procedia-Social and Behavioral Sciences, 30, 639643. doi: 10.1016/j.sbspro.2011.10.124

Renton, A., Phillips, G., Daykin, N., Yu, G., Taylor, K., \& Petticrew, M. (2012). Think of your art-eries: Arts participation, behavioural cardiovascular risk factors and mental well-being in deprived communities in London. Public Health, 126, 557-664. doi: 10.1016/j.puhe.2012.05.025

Reynolds, M. W., Nabors, L., \& Quinlan, A. (2000). The effectiveness of art therapy: Does it work? Art Therapy: Journal of the American Art Therapy Association, 17(3), 207-213. doi: 10.1080/07421656.2000.10129706

Snir, S., \& Regev, D. (2013). ABI - Art-based Intervention Questionnaire. The Arts in Psychoterapy, 40(3), 338-346. doi: 10.1016/j.aip.2013.06.005

Spandler, H., Secker, J., Kent, L., Hacking, S., \& Shenton, J. (2007). Catching life: The contribution of art initiatives to recovery approaches in mental health. Journal of Psychiatric and Mental Health Nursing, 14(8), 791-799. doi: 10.1111/j.13652850.2007.01174.x

Spector, P. (1992). Summated rating scale construction. An Introduction. Newbury Park, CA: Sage.

Stacey, G., \& Stickley, T. (2010). The meaning of art to people who use mental health services. Perspectives in Public Health, 130(2), 70-77. doi: 10.1177/1466424008094811

Stickley, T., \& Hui, A. (2012). Social prescribing through arts on prescription in a UK city: Participants' perspectives (Part 1). Public Health, 126(7), 574579. doi: 10.1016/j.puhe.2012.04.002

Stiles, W. B., Reynolds, S., Hardy, G. E., Rees, A., Bar- kham, M., \& Shapiro, D. A. (1994). Evaluation and description of psychotherapy sessions by clients using the Session Evaluation Questionnaire and the Session Impacts Scale. Journal of Counseling Psychology, 41(2), 175-185. doi: 10.1037/00220167.41 .2 .175

Tucker, N., \& Treviño, A. L. (2011). An art therapy domestic violence prevention group in Mexico. Journal of Clinical Art Therapy, 1(1), 16-24. Recuperado de http://digitalcommons.lmu.edu/jcat/

Uttley, L., Scope, A., Stevenson, M., Rawdin, A., TaylorBuck, E., Sutton, A., \& Wood, C. (2015). Systematic review and economic modelling of the clinical effectiveness and cost-effectiveness of art therapy among people with non-psychotic mental health disorders. Health Technology Assessment, 19(18), 1-120. doi: 10.3310/hta19180

Warson, E. (2003). From foster care to Indian boarding school: Empowering native youth. En D. Betts (Ed.), Creative art therapies approaches in adoption and foster care: Contemporary strategies for working with individuals and families (pp. 131142). Springfield, IL: Charles C. Thomas. 